\title{
Experimental Models of Rheumatoid Arthritis: Acute and Chronic Pain
}

\author{
Ali-Mohammad Khanizadeh ${ }^{1,2}$, Fariba Karimzadeh ${ }^{3 *}$ \\ ${ }^{1}$ Shefa Neuroscience Research Center, Khatam Alanbia Hospital, Tehran, Iran \\ ${ }^{2}$ Department of Physiology, Iran University of Medical Sciences, Tehran, Iran \\ ${ }^{3}$ Cellular and Molecular Research Center, Iran University of Medical Sciences, Tehran, Iran
}

\section{ABSTRACT}

Introduction: Rheumatoid arthritis is the most common chronic inflammatory autoimmune disease with unknown etiology and pathophysiology with various symptoms, such as pain, hyperalgesia, and edema. Changes in the level of cytokines, such as TNF- $\alpha$, IL- 6 and IL-1 $\beta$, are among mechanisms which have been suggested to cause chronic inflammation and have a key role in the design of experimental models of rheumatoid arthritis. The main experimental models of rheumatoid arthritis are Carageen, CFA, collagen-induced arthritis and Zymosan models. In these models, occurrence of thermal hyperalgesia, mechanical allodynia, and pain-induced motor impairment have been reported. These models assess the effectiveness of medications and the mechanisms of pain relief in rheumatoid arthritis. Conclusion: The use of a valid model of rheumatoid arthritis could help to investigate the pathophysiological mechanisms of pain and improve our studies on appropriate treatments for pain.

\section{Key words:}

1. Arthritis, Rheumatoid

2. Pain

3. Disease

*Corresponding Author: Fariba Karimzadeh

E-mail: Fariba_karimzade@yahoo.com

doi: 10.18869/acadpub.shefa.5.1.98 
مدل هاى آزمايشعاهى آرتريت روماتوئيد: درد حاد و مزمن

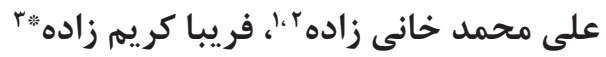

$$
\begin{aligned}
& \text { 'مركز تحقيقات علوم اعصاب شفا، بيمارستان خاتمالانبياء، تهران، ايران }
\end{aligned}
$$

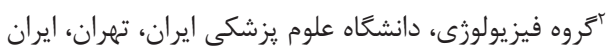

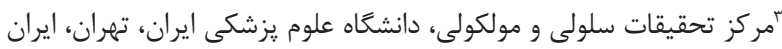

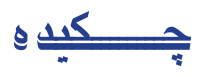

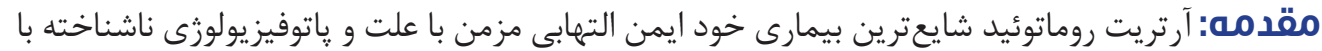

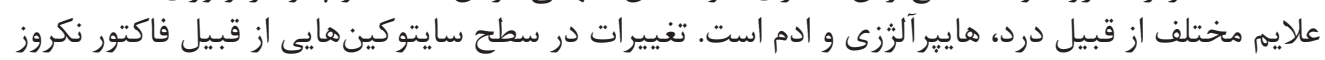

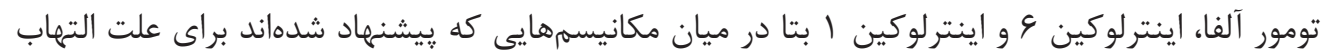

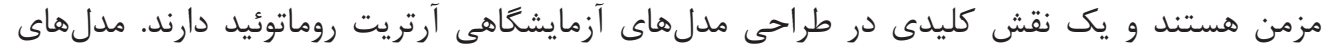

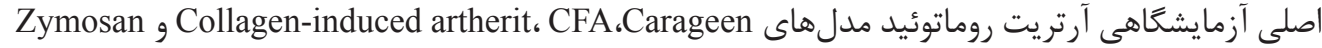

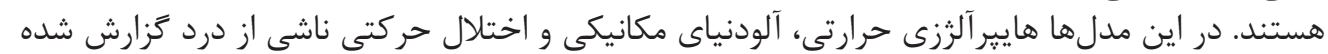

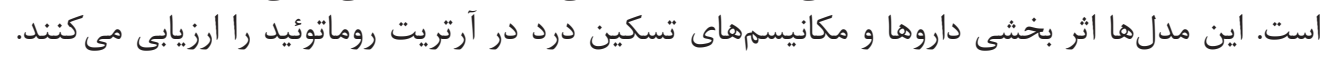

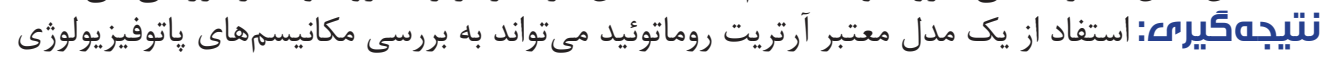
درد و بهبود مطالعات ما در درمانهاى مناسب بران براى درد كمك كند. 


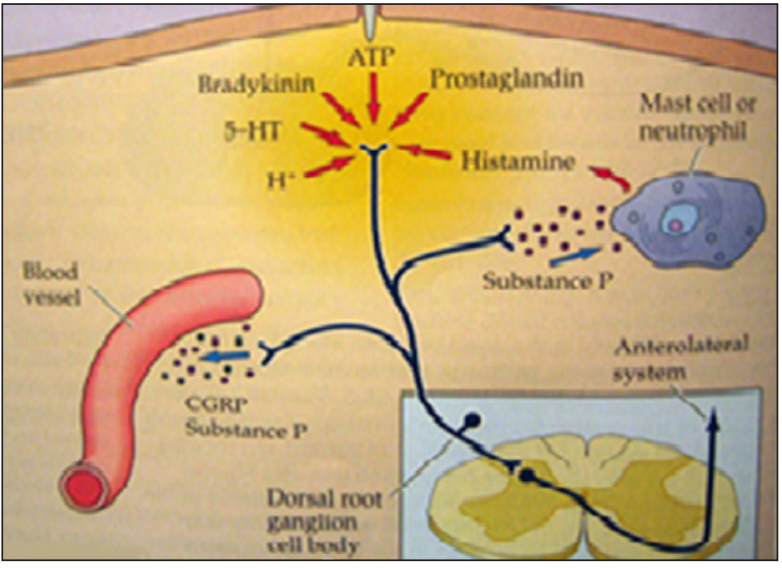

تصوير 1- ايجاد حساسيت محيطى به درد.

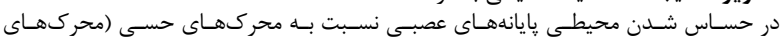

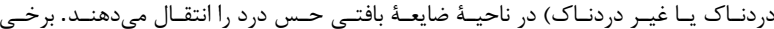

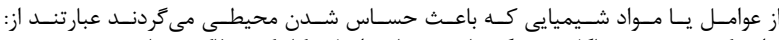

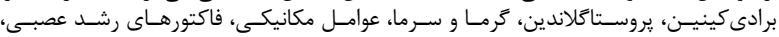

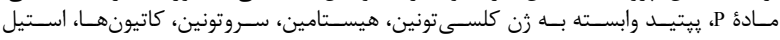

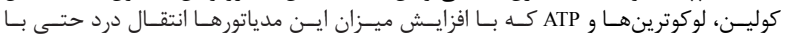

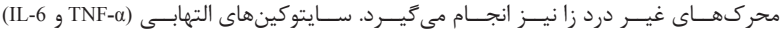

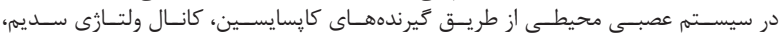

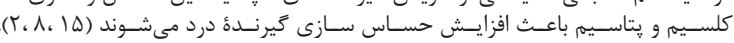

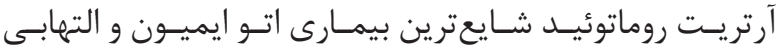

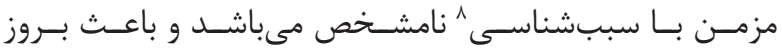

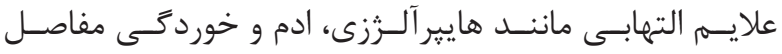

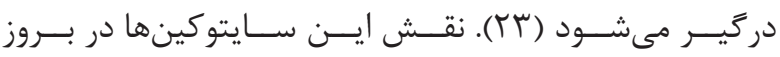

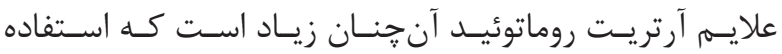

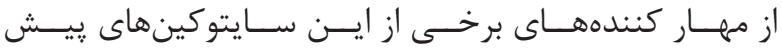

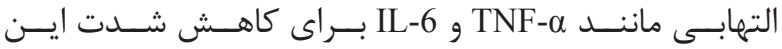

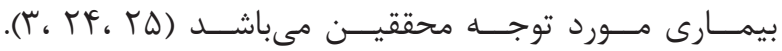

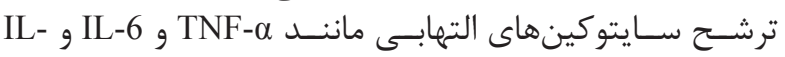

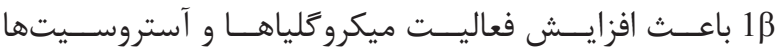

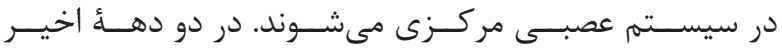

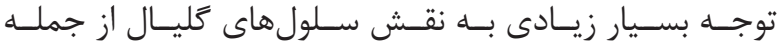

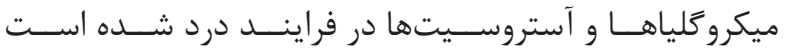

(YG, TV)

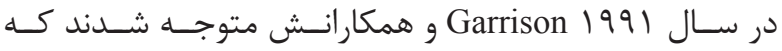

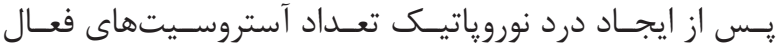

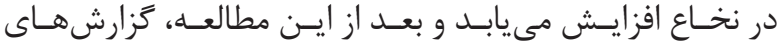

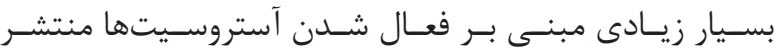

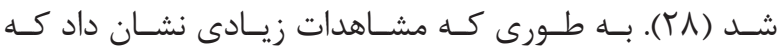

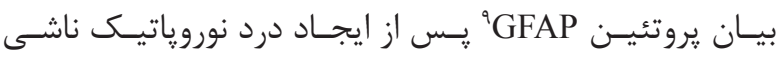

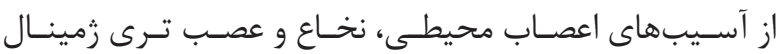

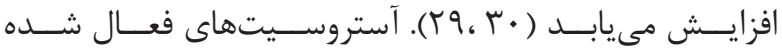

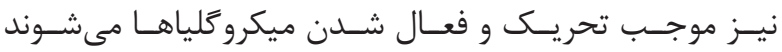

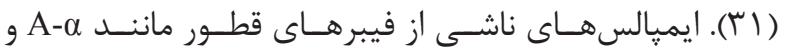

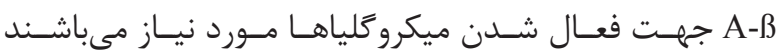

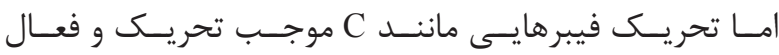

${ }^{1}$ Cytokine

${ }^{2}$ Interleukins

${ }^{3}$ Tumor necrosis factor

${ }^{4}$ Hyperalgesia

${ }^{5}$ Transient receptor potential cation channel subfamily V member

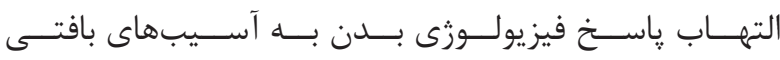

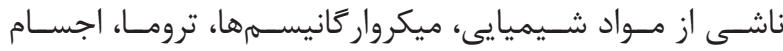

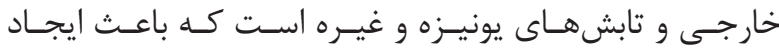

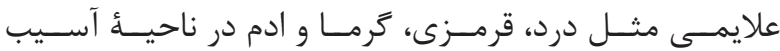

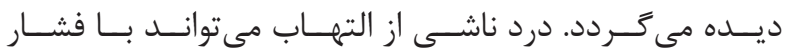

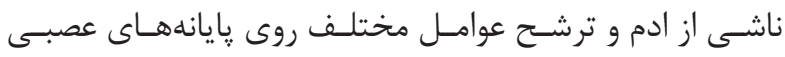

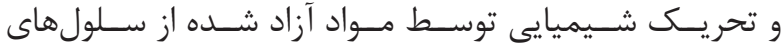

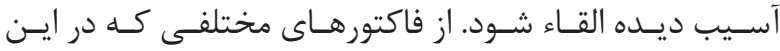

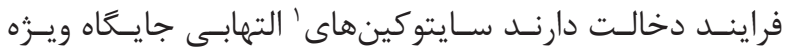

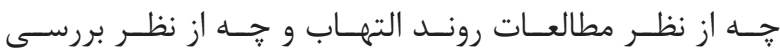

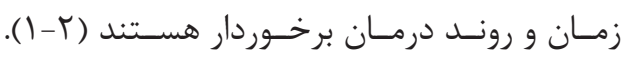

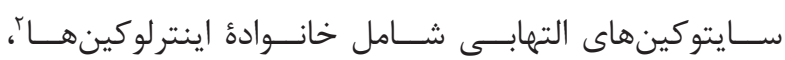

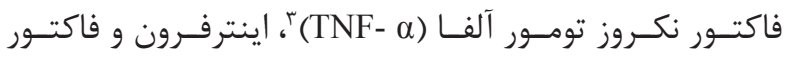

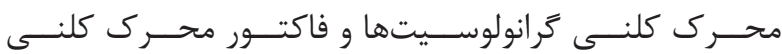

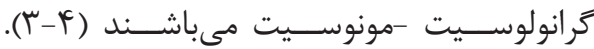

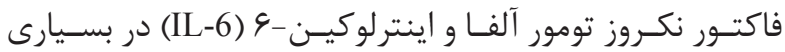

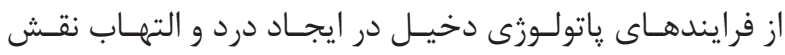

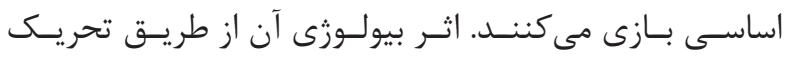

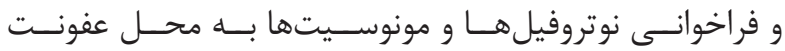

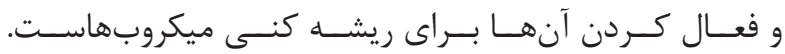

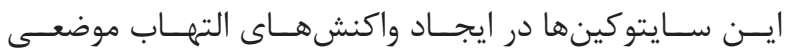

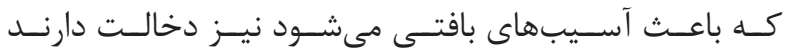

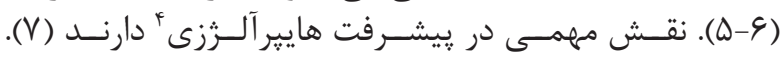

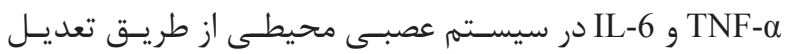

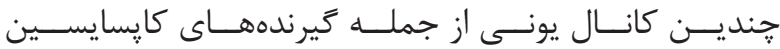

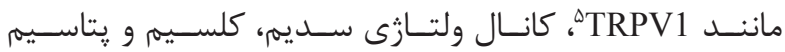

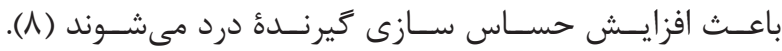

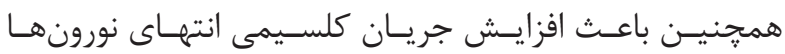

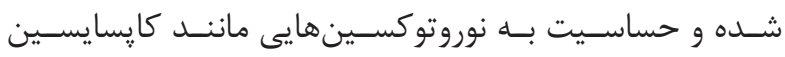

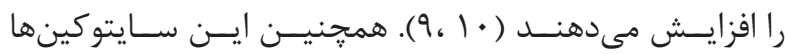

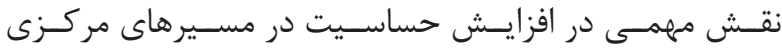

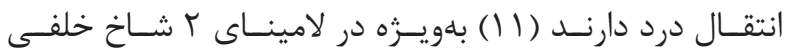

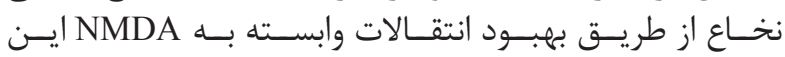

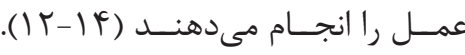

و TNF- $\alpha$

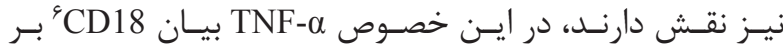

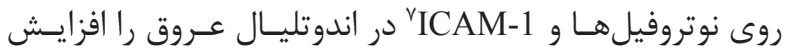

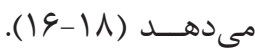

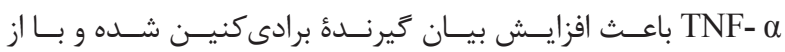

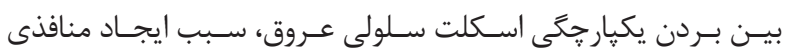

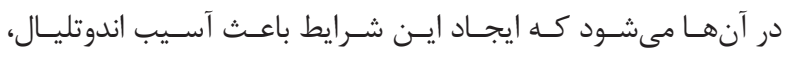

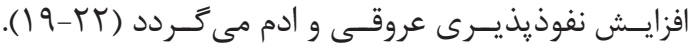

${ }^{6}$ Cluster of differentiation

${ }^{7}$ Intercellular adhesion molecule 1

${ }^{8}$ Etiology

${ }^{9}$ Glial fibrillary acidic protein 


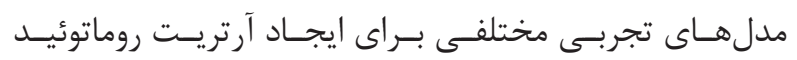

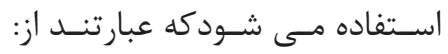

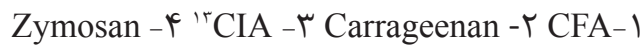

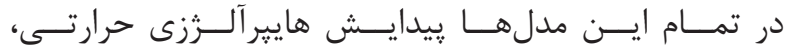

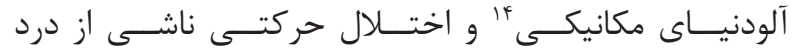

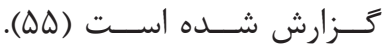

مدل CFA

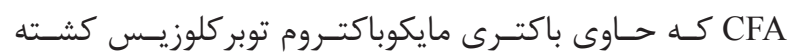

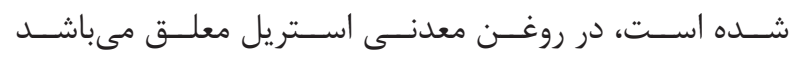

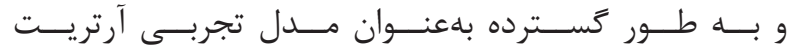

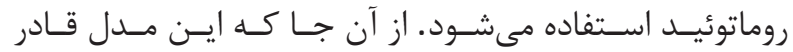

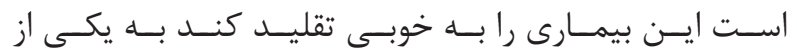

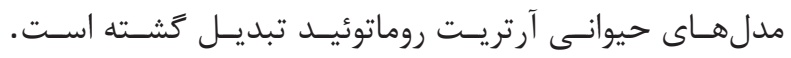

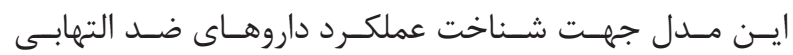

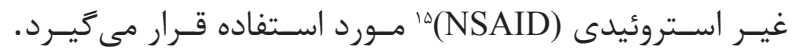

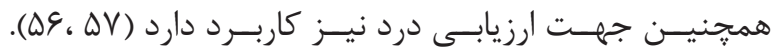

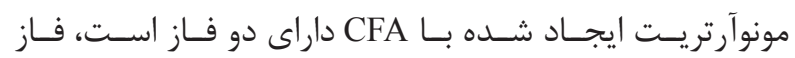

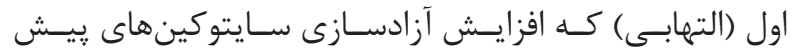

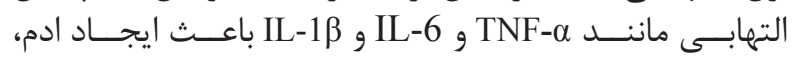

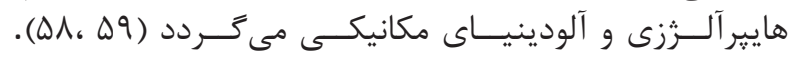

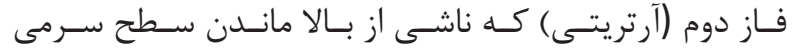

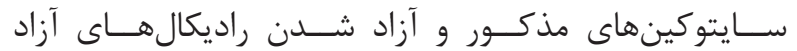

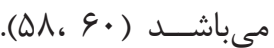

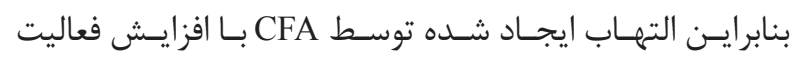

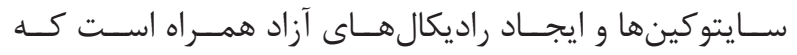

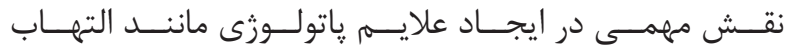

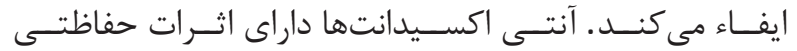

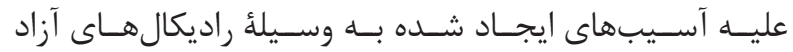

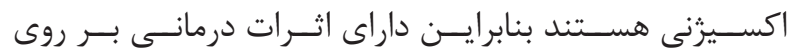

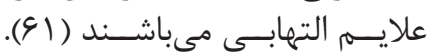

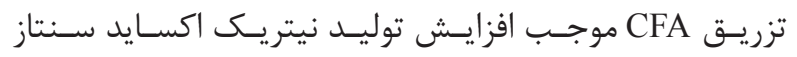

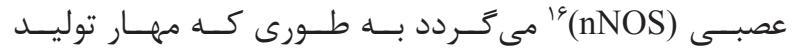

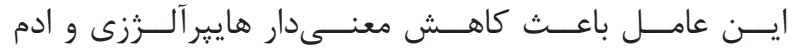

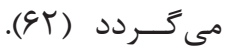

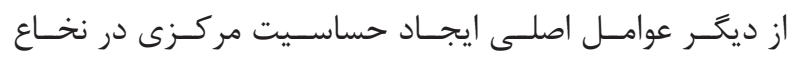

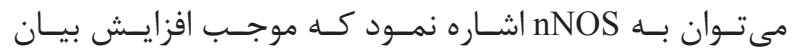

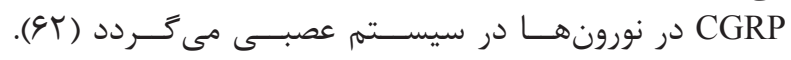

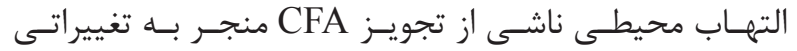

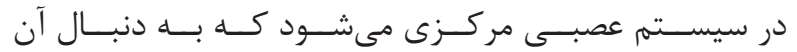

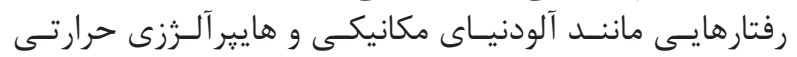

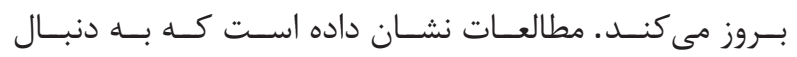

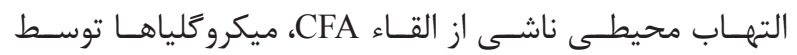

${ }^{10}$ Neurotransmitters

${ }^{11}$ Substance P

${ }^{12}$ Complete freund's adjuvant

${ }^{13}$ Collagen-induced arthritis

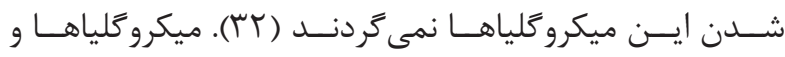

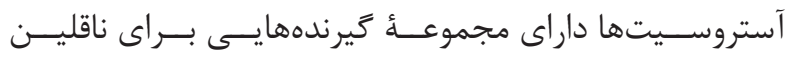

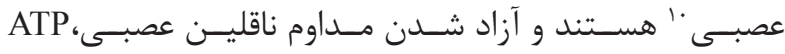

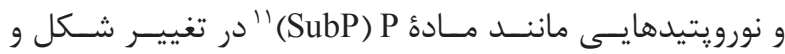

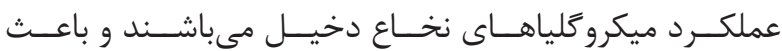

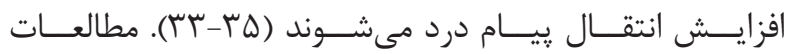

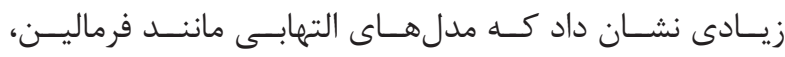

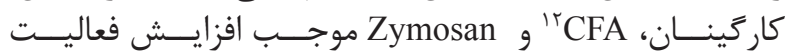

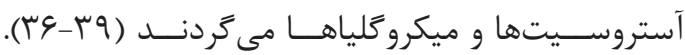

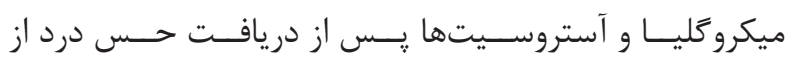

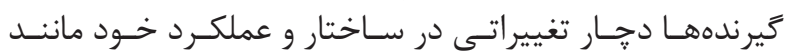

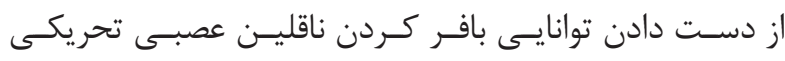

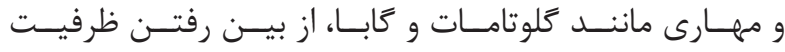

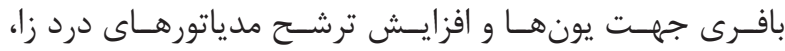

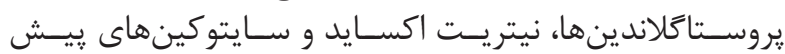

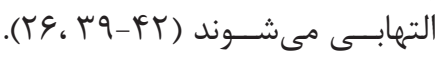

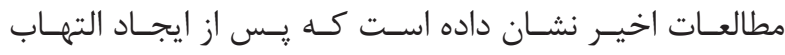

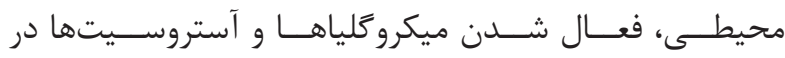

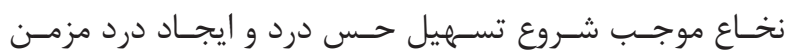

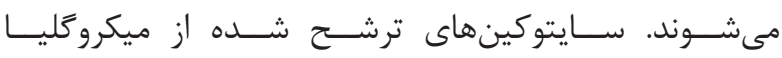

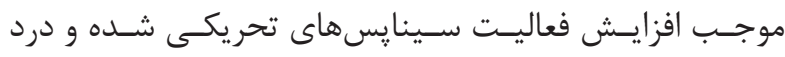

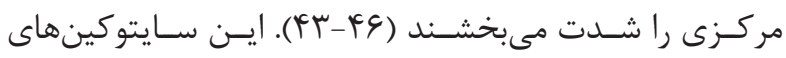

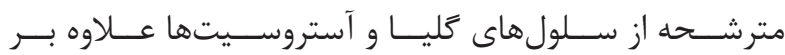

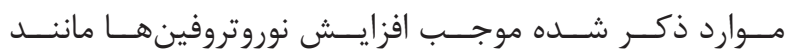

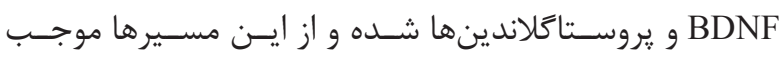

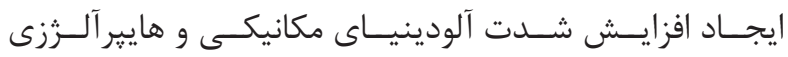

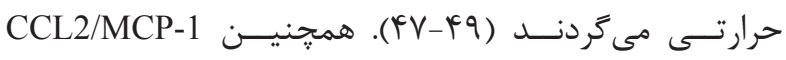

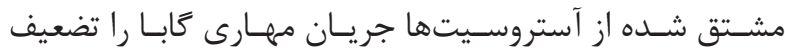

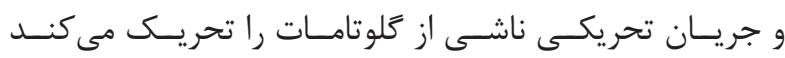

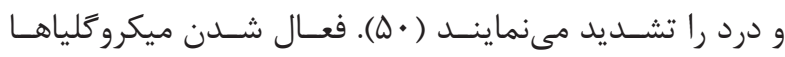

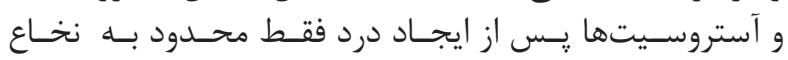

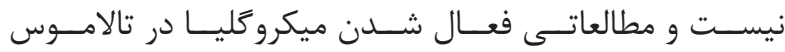

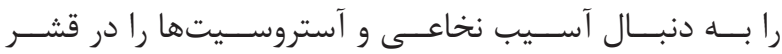

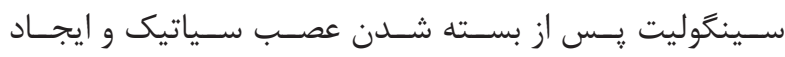

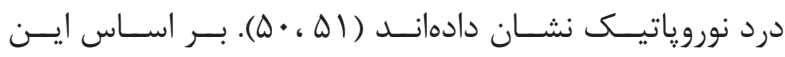

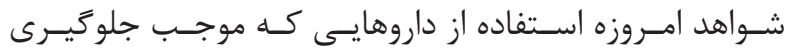

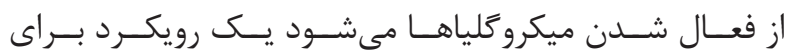

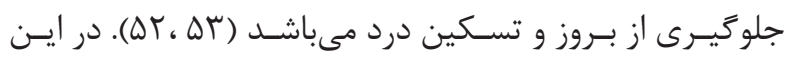

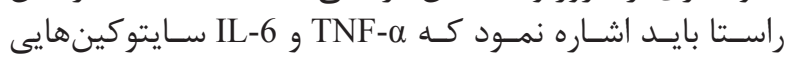

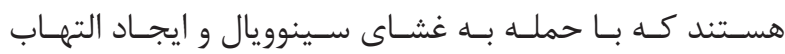

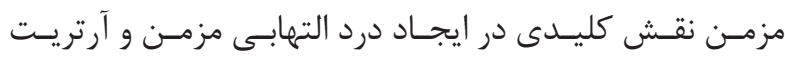

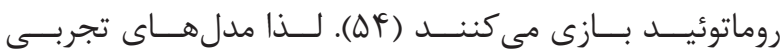

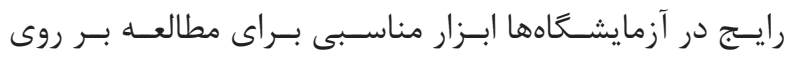

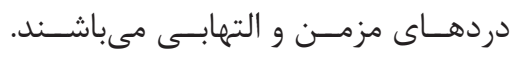

\footnotetext{
${ }^{14}$ Allodynia

${ }^{15}$ Nonsteroidal anti-inflammatory drugs

${ }^{16}$ Neuronal nitric oxide synthases
} 


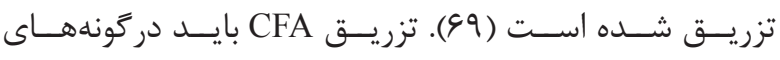

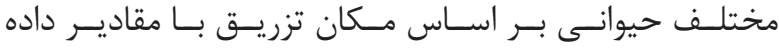

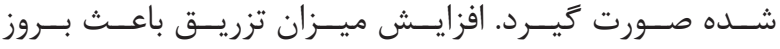

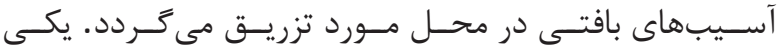

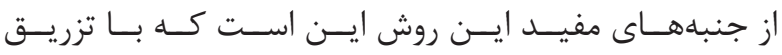

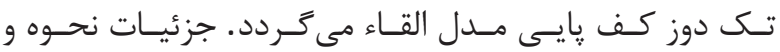

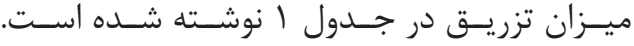

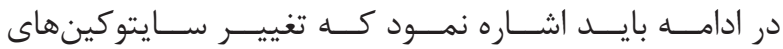

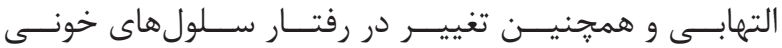

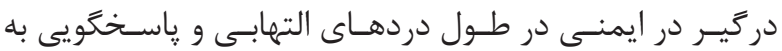

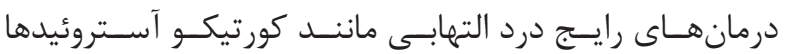

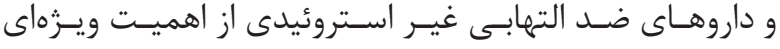

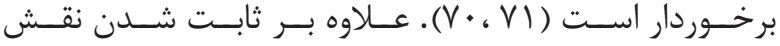

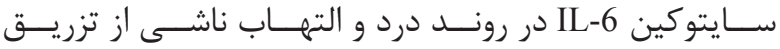

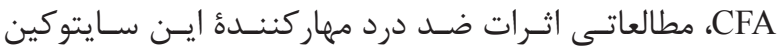

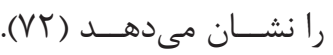

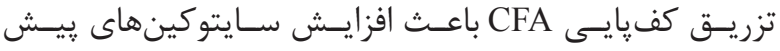

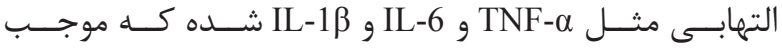

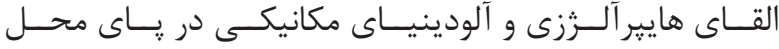

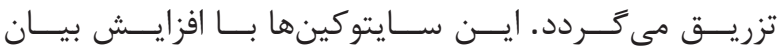

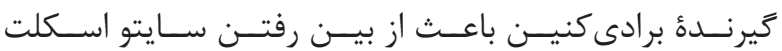

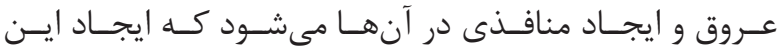

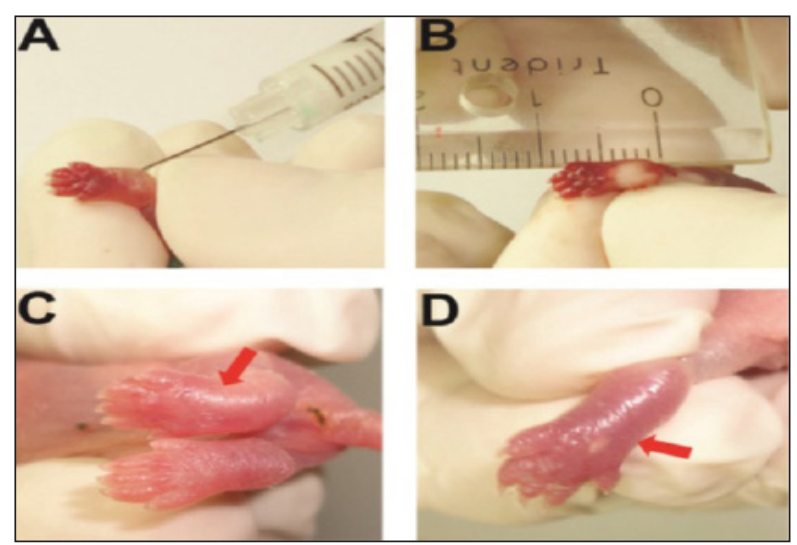

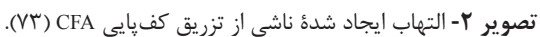

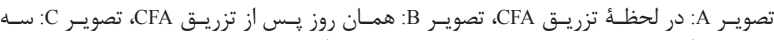

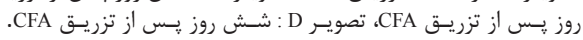

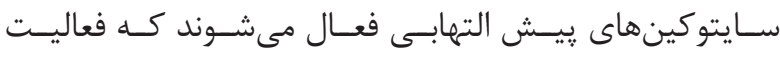

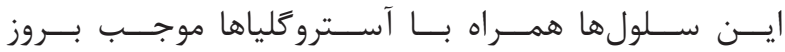

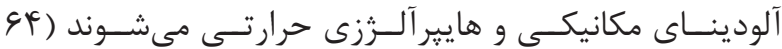

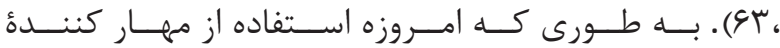

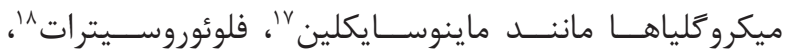

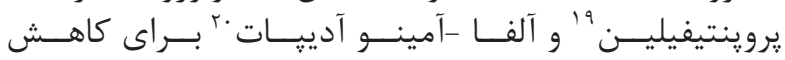

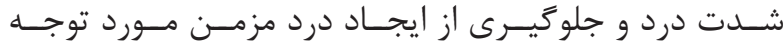

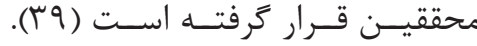

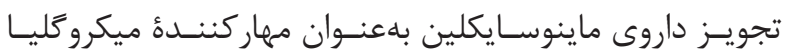

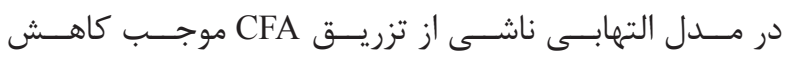

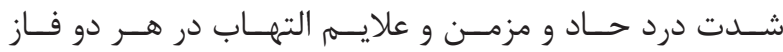

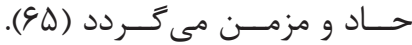

القـاء التهـاب بـا اسـتفاده از CFA باعـث بــروز هايِر آلـرزى از

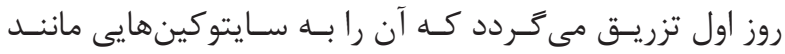

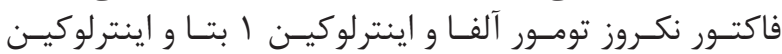

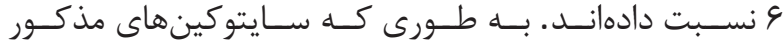

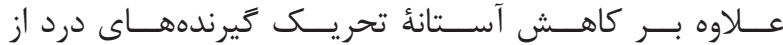

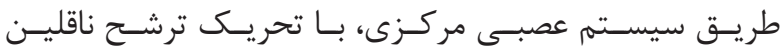

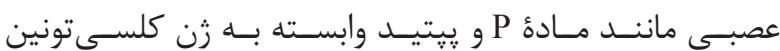
(CGRP)

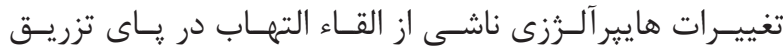

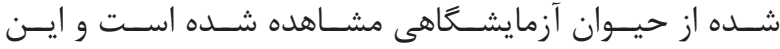

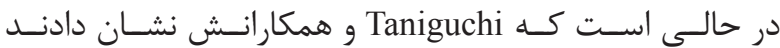

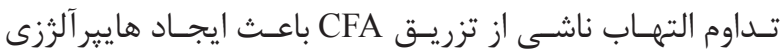

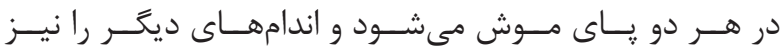

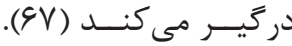

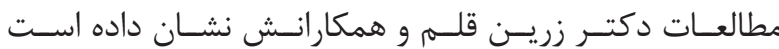

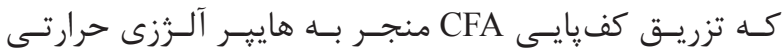

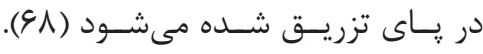

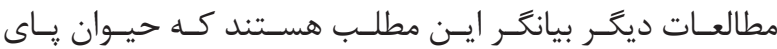

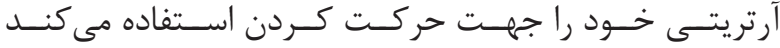

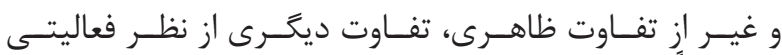

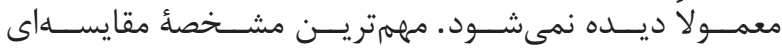

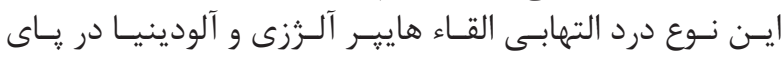

جدول ا- نحوه و ميزان تزريق در مدل CFA.

\begin{tabular}{|c|c|c|c|c|}
\hline كونه & زبو جلدى (mi) & درون جلدى (mI) & قورن صفاقى (mI) & كفيايق (mi) \\
\hline موثُ سورى & $<. / 1$ & - & $<\cdot / \cdot \omega$ & $<\cdot / \cdot \Delta$ \\
\hline موشى صحرابى & $<. / 1$ & $<\cdot 1 \cdot 0$ & $<\cdot 11$ & $<\cdot 11$ \\
\hline خوترون & $<\cdot /{ }_{0}$ & $<\cdot l \cdot \Delta$ & - & $\angle \cdot / r \omega$ \\
\hline
\end{tabular}

${ }^{17}$ Minocycline

${ }^{18}$ Fluorocitrate

${ }^{19}$ Propentophylline
${ }^{20}$ Alpha-aminoadipate

${ }^{21}$ Calcitonin gene related peptide 


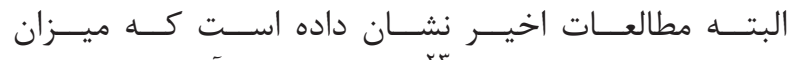
سيكلو اكسـيزناز (COX)

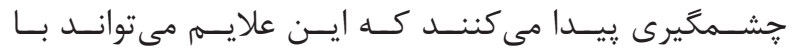

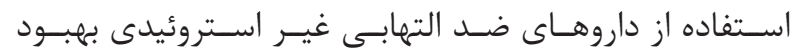

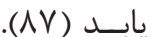

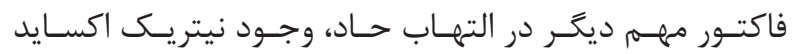

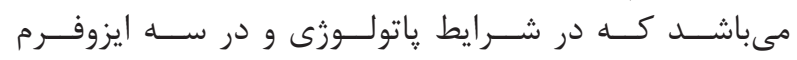

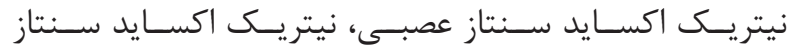

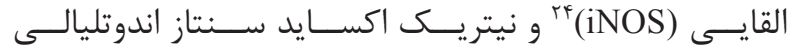

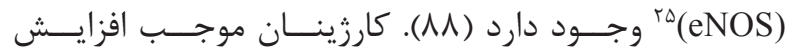

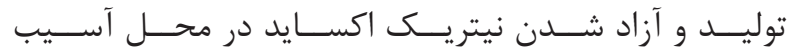

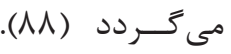

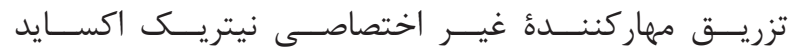

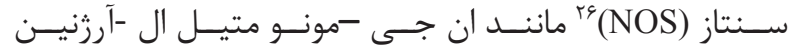

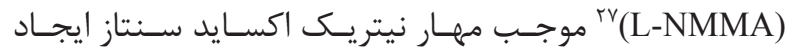

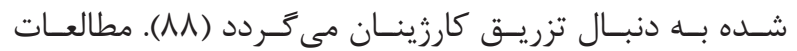

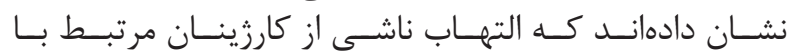

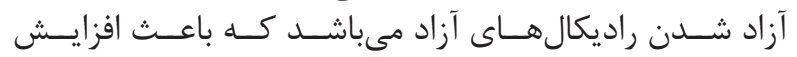

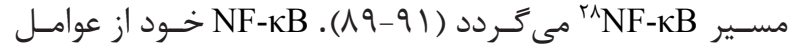

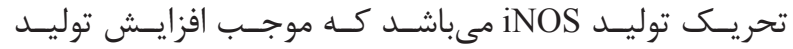

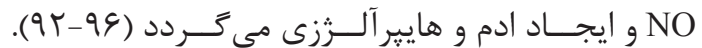

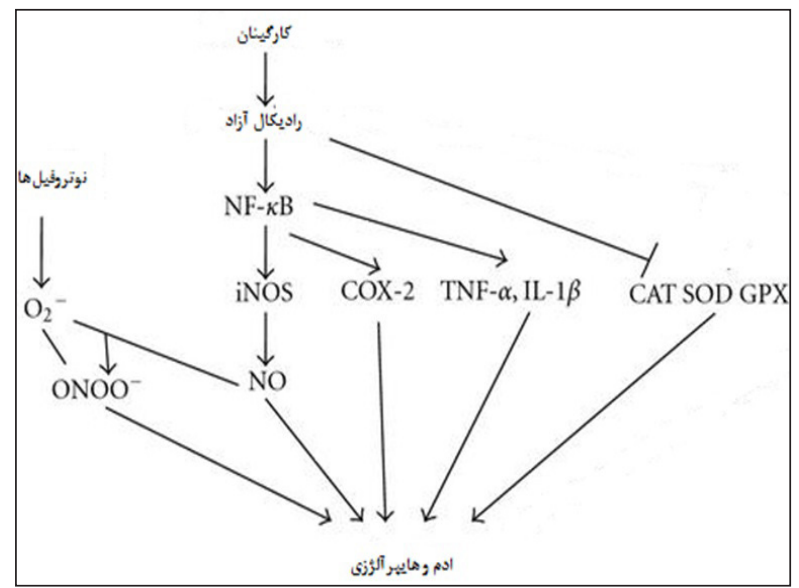

تصوير ץ- روند ايجاد التهاب در مدل كاررينان (qV).

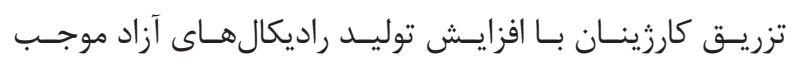

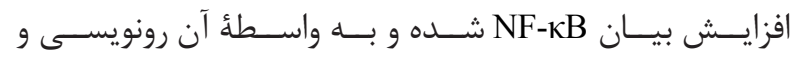

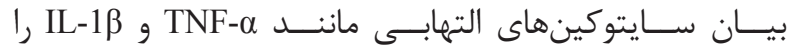

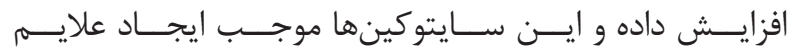

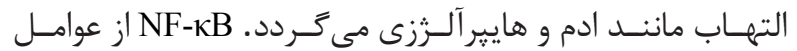

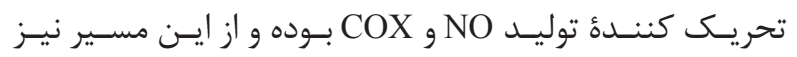

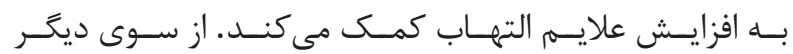

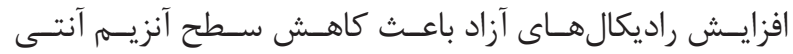

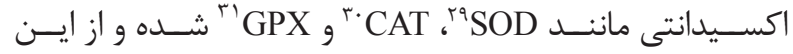

${ }^{22}$ Nitric oxide

${ }^{23}$ Cyclooxygenase

${ }^{24}$ Inducible nitric oxide synthases

${ }^{25}$ Endothelial nitric oxide synthases

${ }^{26}$ Nitric oxide synthases

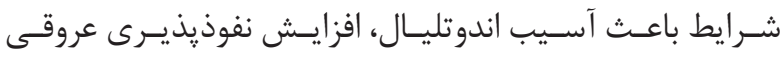

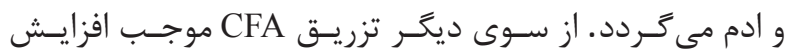

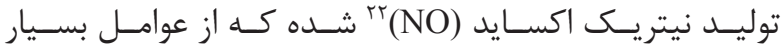

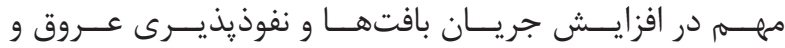

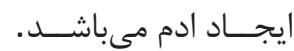

مدل Carrageenan

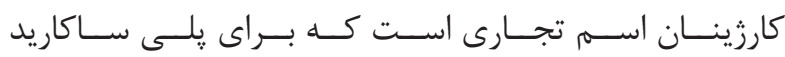

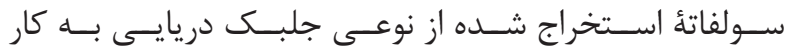

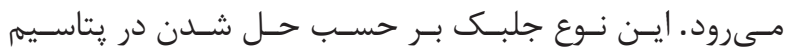

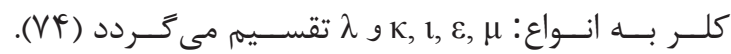

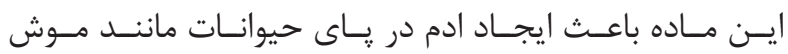

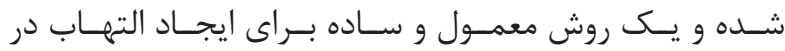

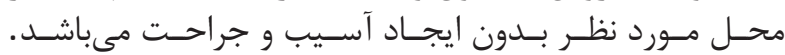

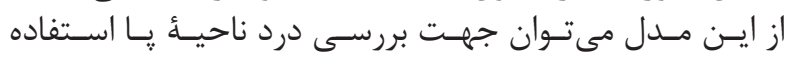

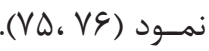

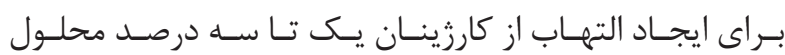

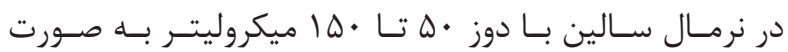

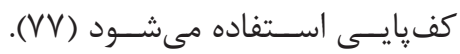

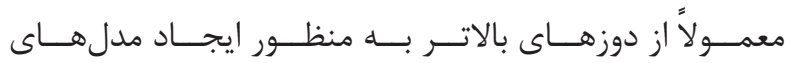

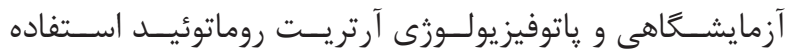

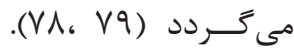

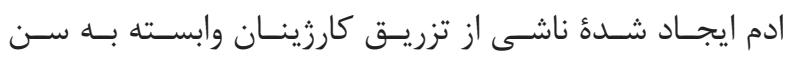

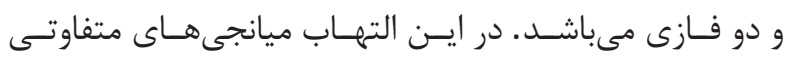

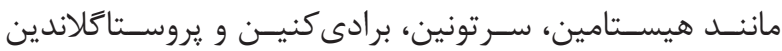

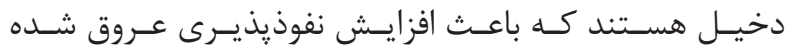

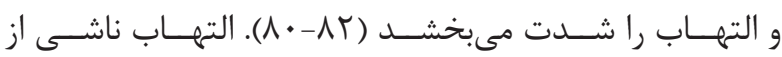

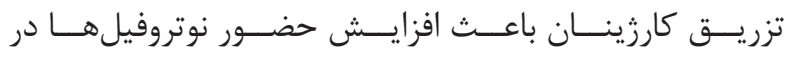

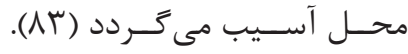

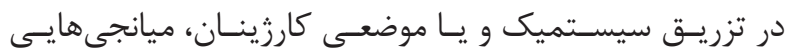

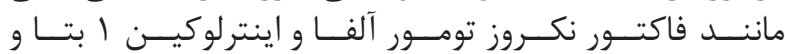

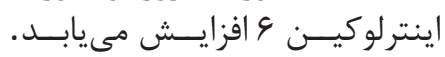

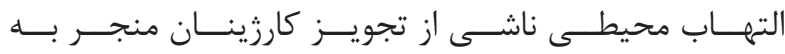

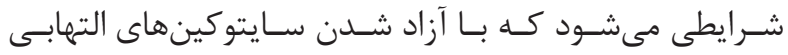

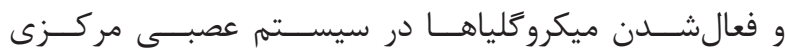

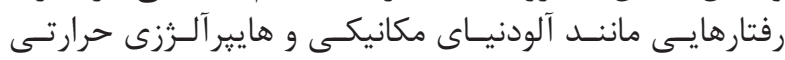

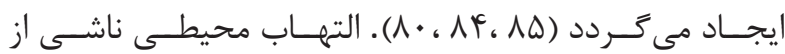

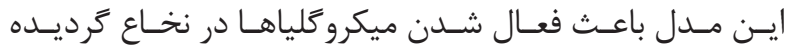

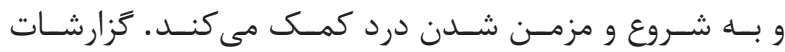

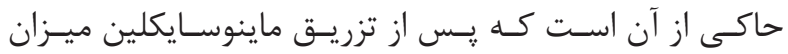

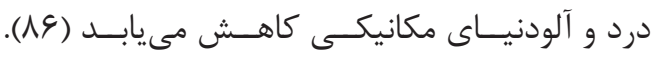

\section{${ }^{27}$ NG-monomethyl L-arginine}

${ }^{28}$ Nuclear factor kappa-light-chain-enhancer of activated B cells

${ }^{29}$ Superoxide dismutase

${ }^{30}$ Catalase

${ }^{31}$ Glutathione peroxidase 


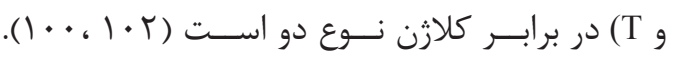

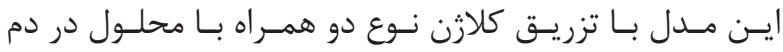

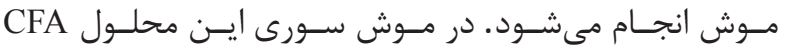

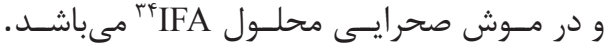

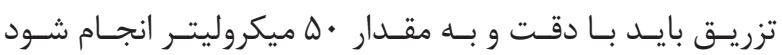

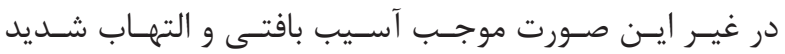

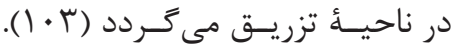

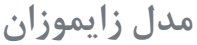

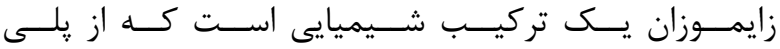

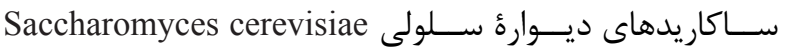

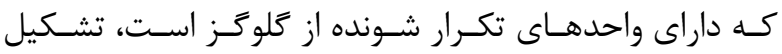

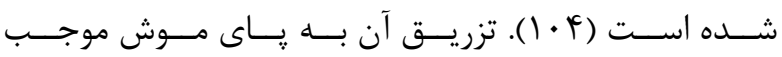

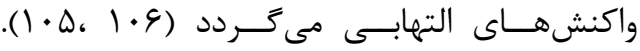

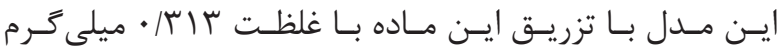

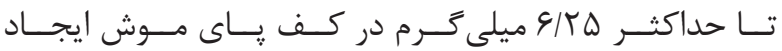

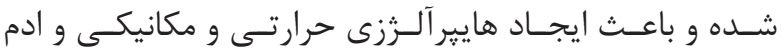

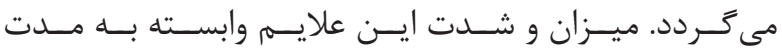

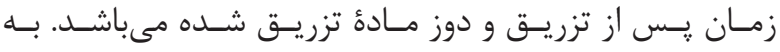

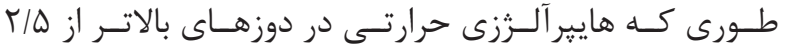

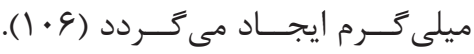

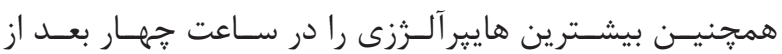

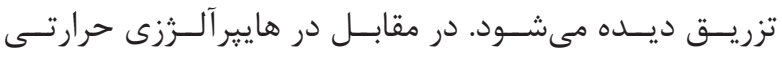

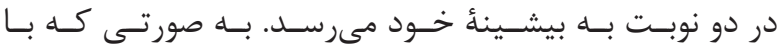

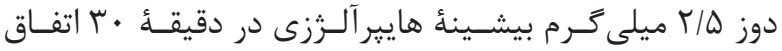

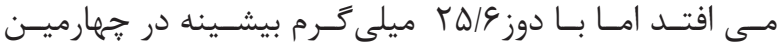

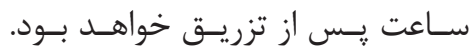

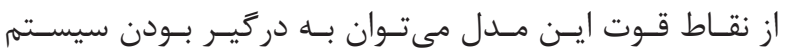
ايمنسى و سـايتوكينها اشـاره نمـود (V • (l).

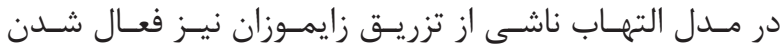

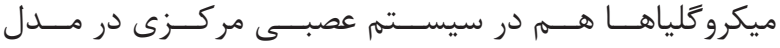

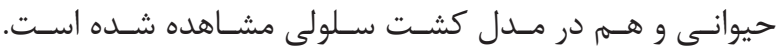

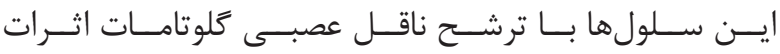

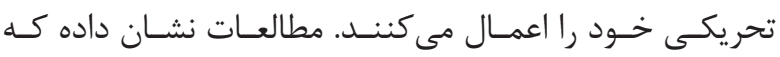

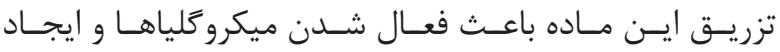

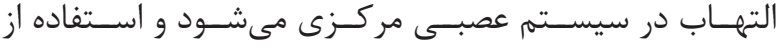

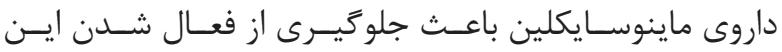

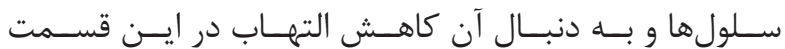

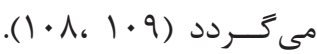

نتيجه

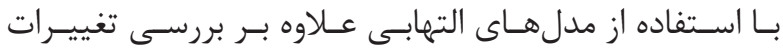

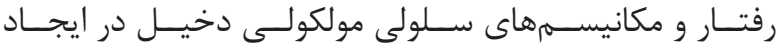

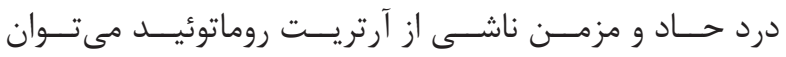

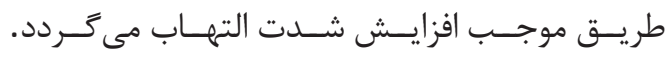

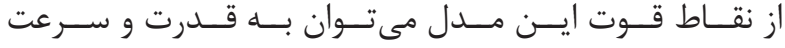

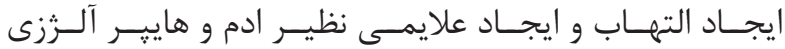

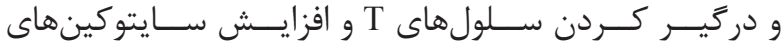

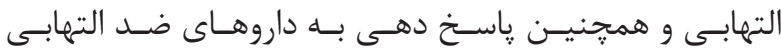

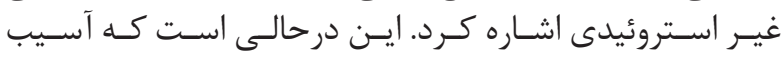

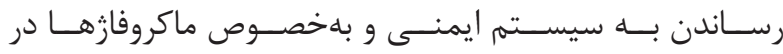

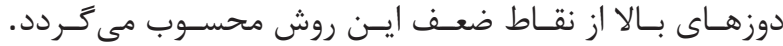

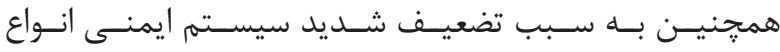

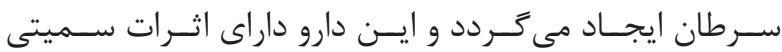

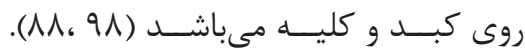

مدل Collagen-Induced Arthritis

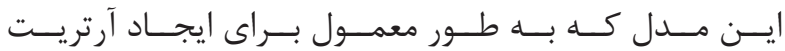

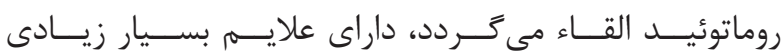

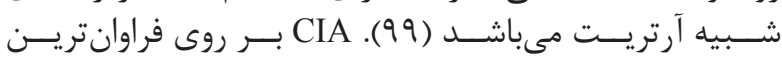

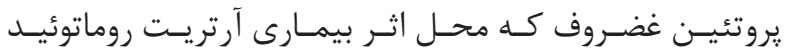

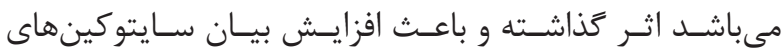

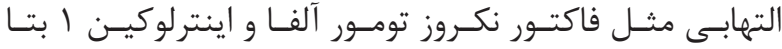

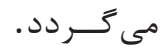

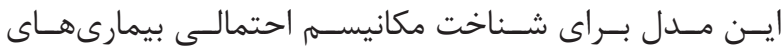

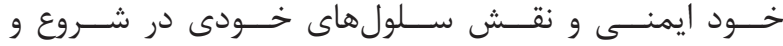

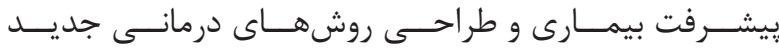

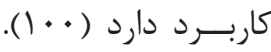

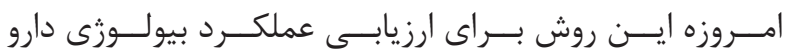

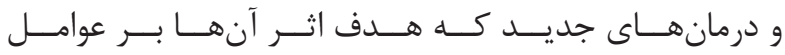

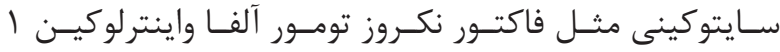

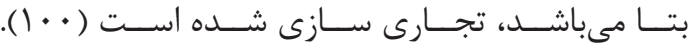

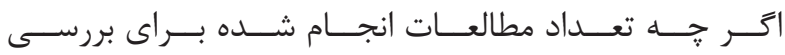

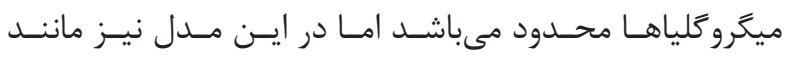

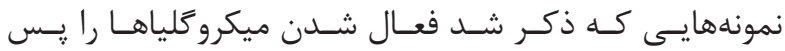

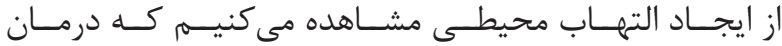

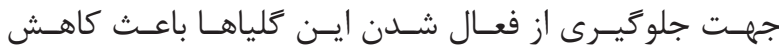

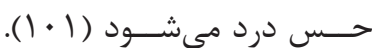

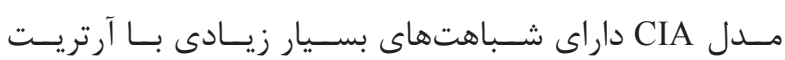

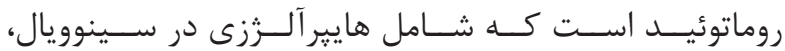

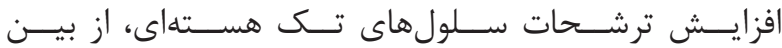

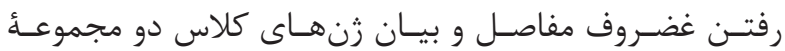

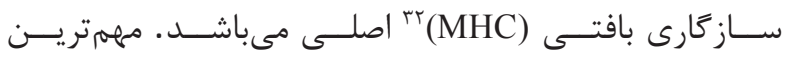

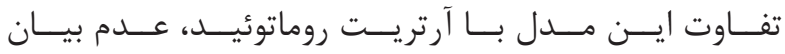

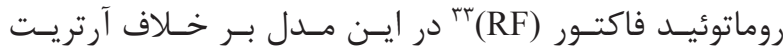

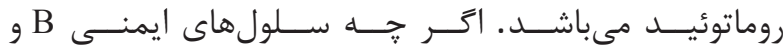

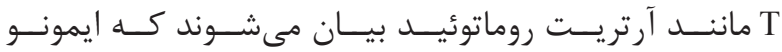

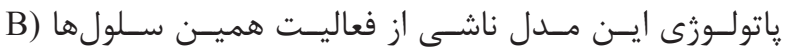

${ }^{32}$ Major histocompatibility complex

${ }^{33}$ Rheumatoid factor

${ }^{34}$ Incomplete freund's adjuvant 


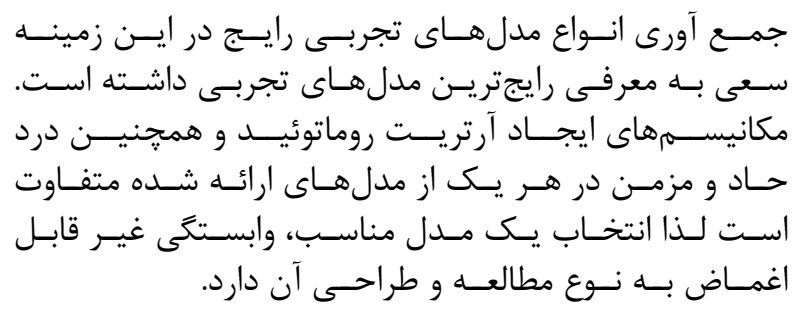

1. Majithia V, Geraci SA. Rheumatoid arthritis: diagnosis and management. Am J Med. 2007; 120(11): 936-9.

2. Ricciotti E, FitzGerald GA. Prostaglandins and inflammation. Arterioscler Thromb Vasc Biol. 2011; 31(5): 986-1000.

3. Hirano T, Matsuda T, Turner M, Miyasaka N, Buchan G, Tang B, et al. Excessive production of interleukin 6/B cell stimulatory factor-2 in rheumatoid arthritis. Eur J Immunol. 1988; 18(11): 1797-802.

4. Zhang J-M, An J. Cytokines, inflammation and pain. Int Anesthesiol Clin. 2007; 45(2): 27.

5. Portenoy RK, Hagen NA. Breakthrough pain: definition, prevalence and characteristics. Pain. 1990; 41(3): 273-81.

6. Kutukculer N, Caglayan S, Aydogdu F. Study of proinflammatory (TNF- $\alpha$, IL-1 $\alpha$, IL-6) and T-cell-derived (IL-2, IL-4) cytokines in plasma and synovial fluid of patients with juvenile chronic arthritis: correlations with clinical and laboratory parameters. J Clin Rheumatol. 1998; 17(4): 288-92.

7. Ahmad SF, Ansari MA, Zoheir KM, Bakheet SA, Korashy HM, Nadeem A, et al. Regulation of TNF- $\alpha$ and NF- $\kappa$ B activation through the JAK/STAT signaling pathway downstream of histamine 4 receptor in a rat model of LPS-induced joint inflammation. J Immunol. 2015; 220(7): 889-98.

8. Park C-K, Lü N, Xu Z-Z, Liu T, Serhan CN, Ji R-R. Resolving TRPV1-and TNF- $\alpha$-mediated spinal cord synaptic plasticity and inflammatory pain with neuroprotectin D1. J Neurosci. 2011; 31(42): 15072-85.

9. Khan A, Diogenes A, Jeske N, Henry M, Akopian A, Hargreaves K. Tumor necrosis factor $\alpha$ enhances the sensitivity of rat trigeminal neurons to capsaicin. Neuroscience. 2008; 155(2): 503-9.

10. Sappington RM, Calkins DJ. Contribution of TRPV1 to microglia-derived IL-6 and NFאB translocation with elevated hydrostatic pressure. Invest Ophthalmol Vis. 2008; 49(7): 3004-17.

11. Kawasaki Y, Zhang L, Cheng J-K, Ji R-R. Cytokine

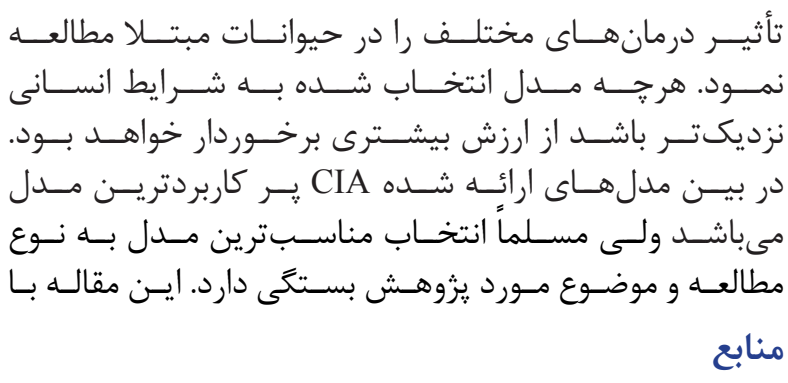

mechanisms of central sensitization: distinct and overlapping role of interleukin-1 $\beta$, interleukin-6, and tumor necrosis factor- $\alpha$ in regulating synaptic and neuronal activity in the superficial spinal cord. $\mathrm{J}$ Neurosci. 2008; 28(20): 5189-94.

12. 菊池宏信. Thermo-sensitive transient receptor potential vanilloid (trpv) channels in mouse adipocytes. University of Tokyo. 2014. http://hdl.handle. net/2261/58150.

13. Kikuchi H, Oguri G, Yamamoto Y, Takano N, Tanaka $\mathrm{T}$, Takahashi $\mathrm{M}$, et al. Thermo-Sensitive transient receptor potential vanilloid (trpv) channels regulate il-6 expression in mouse adipocytes. J Cardiovasc Pharmacol. 2015; 4(4).

14. Erta M, Quintana A, Hidalgo J. Interleukin-6, a major cytokine in the central nervous system. Int J Biol Sci. 2012; 8(9): 1254-66.

15. Proprioceptor, cutaneous receptor and pain receptor. http:/katie humanbio. blogspot.com/2008/04/ proprioceptors - cutaneous - receptors - pain.html.

16. Lo SK, Everitt J, Gu J, Malik AB. Tumor necrosis factor mediates experimental pulmonary edema by ICAM-1 and CD18-dependent mechanisms. J Clin Invest. 1992; 89(3): 981.

17. Chandrasekharan UM, Siemionow M, Unsal M, Yang L, Poptic E, Bohn J, et al. Tumor necrosis factor $\alpha(\mathrm{TNF}-\alpha)$ receptor-II is required for TNF- $\alpha$-induced leukocyte-endothelial interaction in vivo. Blood. 2007; 109(5): 1938-44.

18. Maruo N, Morita I, Shirao M, Murota S. IL-6 increases endothelial permeability in vitro. J Endocrinol. 1992; 131(2): 710-4.

19. Campos MM, Souza GE, Calixto JB. Modulation of kinin B 1 but not B 2 receptors-mediated rat paw edema by IL-1 $\beta$ and TNF $\alpha$. Peptides. 1998; 19(7): 1269-76.

20. Chappell D, Hofmann-Kiefer K, Jacob M, Rehm M, Briegel J, Welsch U, et al. TNF- $\alpha$ induced shedding of the endothelial glycocalyx is prevented by hydrocortisone and antithrombin. Basic Res Cardiol. 2009; 104(1): 78-89. 
21. Goldblum SE, Sun WL. Tumor necrosis factor-alpha augments pulmonary arterial transendothelial albumin flux in vitro. Am J Physiol. 1990; 258(2): L57-L67.

22. Angelini DJ, Hyun S-W, Grigoryev DN, Garg P, Gong P, Singh IS, et al. TNF- $\alpha$ increases tyrosine phosphorylation of vascular endothelial cadherin and opens the paracellular pathway through fyn activation in human lung endothelia. Am J Physiol Lung Cell Mol Physiol. 2006; 291(6): L1232-L45.

23. Di Giovine FS, Nuki G, Duff GW. Tumour necrosis factor in synovial exudates. Ann Rheum Dis. 1988; 47(9): 768-72.

24. Ma X, Xu S. TNF inhibitor therapy for rheumatoid arthritis (Review). Biomed Rep. 2013; 1(2): 177-84.

25. Woodrick R, Ruderman EM. Anti-interleukin-6 therapy in rheumatoid arthritis. Bull NYU Hosp Jt Dis. 2010; 68(3): 211-7.

26. Milligan ED, Watkins LR. Pathological and protective roles of glia in chronic pain. Nat. Rev. Neurosci. 2009; 10(1): 23-36.

27. Scholz J, Woolf CJ. The neuropathic pain triad: neurons, immune cells and glia. Nat Neurosci. 2007; 10(11): 1361-8.

28. Garrison C, Dougherty P, Kajander K, Carlton S. Staining of glial fibrillary acidic protein (GFAP) in lumbar spinal cord increases following a sciatic nerve constriction injury. Brain Res. 1991; 565(1): 1-7.

29. Vega-Avelaira D, Moss A, Fitzgerald M. Age-related changes in the spinal cord microglial and astrocytic response profile to nerve injury. Brain Behav. 2007; 21(5): $617-23$.

30. Stuesse S, Crisp T, McBurney D, Schechter J, Lovell J, Cruce W. Neuropathic pain in aged rats: behavioral responses and astrocytic activation. Experimental Brain Research. 2001; 137(2): 219-27.

31. Röhl C, Lucius R, Sievers J. The effect of activated microglia on astrogliosis parameters in astrocyte cultures. Brain Res. 2007; 1129: 43-52.

32. Suter MR, Berta T, Gao Y-J, Decosterd I, Ji R-R. Large a-fiber activity is required for microglial proliferation and p38 MAPK activation in the spinal cord: different effects of resiniferatoxin and bupivacaine on spinal microglial changes after spared nerve injury. Mol Pain. 2009; 5: 53. doi: 10.1186/1744-8069-5-53.

33. D’Antoni S, Berretta A, Bonaccorso CM, Bruno V,
Aronica E, Nicoletti F, et al. Metabotropic glutamate receptors in glial cells. Neurochem Res. 2008; 33(12): 2436-43.

34. Masmoudi-Kouki O, Gandolfo P, Leprince J, Vaudry D, Pelletier G, Fournier A, et al. PACAP stimulates biosynthesis and release of endozepines from rat astrocytes. Ann N. Y Acad Sci. 2006; 1070: 411-6.

35. Gosselin R, Dansereau M, Pohl M, Kitabgi P, Beaudet N, Sarret P, et al. Chemokine network in the nervous system: a new target for pain relief. Curr Med Chem. 2008; 15(27): 2866-75.

36. Shan S, Hong C, Mei H, Ting-Ting L, Hai-Li P, ZhiQi Z, et al. New evidence for the involvement of spinal fractalkine receptor in pain facilitation and spinal glial activation in rat model of monoarthritis. Pain. 2007; 129(1): 64-75.

37. Sweitzer S, Colburn R, Rutkowski M, DeLeo J. Acute peripheral inflammation induces moderate glial activation and spinal IL-1 $\beta$ expression that correlates with pain behavior in the rat. Brain Res. 1999; 829(1): 209-21.

38. Sun Y-N, Luo J-Y, Rao Z-R, Lan L, Duan L. GFAP and Fos immunoreactivity in lumbo-sacral spinal cord and medulla oblongata after chronic colonic inflammation in rats. World J Gastroenterol. 2005; 11(31): 4827-32.

39. Gosselin R-D, Suter MR, Ji R-R, Decosterd I. Glial cells and chronic pain. Neuroscientist. 2010; 16(5): 519-31.

40. Watkins LR, Milligan ED, Maier SF. Glial activation: a driving force for pathological pain. Trends Neurosci. 2001; 24(8): 450-5.

41. Ghorbani Gazar S, Talebi F. Immune System in central nervous system. Shefaye Khatam. 2013; 1(1): 49-54.

42. Sajadian A, Jafarian M, Khodaie B, Mohammad Sadeghi S, Ghaemi A. Reduction of neuroinflammation in epilepsy by using induced pluripotent stem (ips) cellsderived astrocytes. Shefaye Khatam. 2014; 2(2): 56-64.

43. Xu J-T, Xin W-J, Zang Y, Wu C-Y, Liu X-G. The role of tumor necrosis factor-alpha in the neuropathic pain induced by Lumbar 5 ventral root transection in rat. Pain. 2006; 123(3): 306-21.

44. Stohwasser R, Giesebrecht J, Kraft R, Müller EC, Häusler KG, Kettenmann $\mathrm{H}$, et al. Biochemical analysis of proteasomes from mouse microglia: Induction of immunoproteasomes by interferon- $\gamma$ and 
lipopolysaccharide. Glia. 2000; 29(4): 355-65.

45. Breder CD, Tsujimoto M, Terano Y, Scott DW, Saper $\mathrm{CB}$. Distribution and characterization of tumor necrosis factor- $\alpha$-like immunoreactivity in the murine central nervous system. J Comp Neurol. 1993; 337(4): 543-67.

46. Woolf CJ, Salter MW. Neuronal plasticity: increasing the gain in pain. Science. 2000; 288(5472): 1765-8.

47. Li C-Q, Xu J-M, Liu D, Zhang J-Y, Dai R-P. Brain derived neurotrophic factor (BDNF) contributes to the pain hypersensitivity following surgical incision in the rats. Mol Pain. 2008; 4: 27. doi: 10.1186/1744-80694-27.

48. Lu VB, Biggs JE, Stebbing MJ, Balasubramanyan $\mathrm{S}$, Todd KG, Lai AY, et al. Brain-derived neurotrophic factor drives the changes in excitatory synaptic transmission in the rat superficial dorsal horn that follow sciatic nerve injury. J Physiol. 2009; 587(5): 1013-32.

49. McMahon SB, Cafferty WB, Marchand F. Immune and glial cell factors as pain mediators and modulators. Exp Neurol. 2005; 192(2): 444-62.

50. Gao Y-J, Zhang L, Samad OA, Suter MR, Yasuhiko K, $\mathrm{Xu} \mathrm{Z}-\mathrm{Z}$, et al. JNK-induced MCP-1 production in spinal cord astrocytes contributes to central sensitization and neuropathic pain. J Neurosci. 2009; 29(13): 4096-108.

51. Kuzumaki N, Narita M, Hareyama N, Niikura $\mathrm{K}$, Nagumo Y, et al. Chronic pain-induced astrocyte activation in the cingulate cortex with no change in neural or glial differentiation from neural stem cells in mice. Neurosci Lett. 2007; 415(1): 22-7.

52. Milligan ED, Twining C, Chacur M, Biedenkapp J, O'Connor K, Poole S, et al. Spinal glia and proinflammatory cytokines mediate mirror-image neuropathic pain in rats. J Neurosci. 2003; 23(3): 1026-40.

53. Svensson CI, Marsala M, Westerlund A, Calcutt NA, Campana WM, Freshwater JD, et al. Activation of p38 mitogen-activated protein kinase in spinal microglia is a critical link in inflammation-induced spinal pain processing. J Neurochem. 2003; 86(6): 1534-44.

54. Inglis JJ, Nissim A, Lees DM, Hunt SP, Chernajovsky Y, Kidd BL. The differential contribution of tumour necrosis factor to thermal and mechanical hyperalgesia during chronic inflammation. Arthritis Res Ther. 2005; 7(4): R807-16.

55. Nagakura Y, Okada M, Kohara A, Kiso T, Toya T, Iwai A, et al. Allodynia and hyperalgesia in adjuvantinduced arthritic rats: time course of progression and efficacy of analgesics. J Pharm Exp Ther. 2003; 306(2): 490-7.

56. Philippe L, Gegout-Pottie P, Guingamp C, Bordji K, Terlain B, Netter P, et al. Relations between functional, inflammatory, and degenerative parameters during adjuvant arthritis in rats. Am J Physiol Regul Integr Comp Physiol. 1997; 273(4): R1550-R6.

57. Billiau A, Matthys P. Modes of action of Freund's adjuvants in experimental models of autoimmune diseases. J Leukoc Biol. 2001; 70(6): 849-60.

58. Zaringhalam J, Akbari A, Tekieh E, Manaheji $\mathrm{H}$, Rezazadeh S. Achillea santolina reduced serum IL-6 level and hyperalgesia during CFA-induced inflammation in male Wistar rats. J Chin Integr Med. 2010; 12: 1180-9.

59. Boyce-Rustay JM, Zhong C, Kohnken R, Baker SJ, Simler GH, Wensink EJ, et al. Comparison of mechanical allodynia and the affective component of inflammatory pain in rats. Neuropharmacol. 2010; 58(2): 537-43.

60. Rodriguez-Vita J, Lawrence T. The resolution of inflammation and cancer. Cytokine Growth Factor Rev. 2010; 21(1): 61-5.

61. Fraser GL, Gaudreau GA, Clarke P, Ménard DP, Perkins MN. Antihyperalgesic effects of $\delta$ opioid agonists in a rat model of chronic inflammation. Br J Pharmacol. 2000; 129(8): 1668-72.

62. Boettger MK, Üceyler N, Zelenka M, Schmitt A, Reif A, Chen Y, et al. Differences in inflammatory pain in nNOS-, iNOS-and eNOS-deficient mice. Eur J Pain. 2007; 11(7): 810-8.

63. Raghavendra V, Tanga FY, DeLeo JA. Complete freunds adjuvant-induced peripheral inflammation evokes glial activation and proinflammatory cytokine expression in the CNS. Eur J Neurosci. 2004; 20(2): 467-73.

64. Clark AK, Gentry C, Bradbury EJ, McMahon SB, Malcangio M. Role of spinal microglia in rat models of peripheral nerve injury and inflammation. Eur J Pain. 2007; 11(2): 223-30.

65. Nasseri B, Nazemian V, Manaheji H, Zaringhalam $\mathrm{J}$. Microglia are involve in pain related behaviors during the acute and chronic phase of arthritis inflammation. J Cell Mol Anesth. 2016; 1(4): 137-45.

66. Cahill C, Morinville A, Hoffert C, O'Donnell D, Beaudet A. Up-regulation and trafficking of $\delta$ opioid receptor in a model of chronic inflammation: implications 


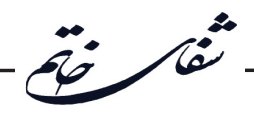

for pain control. Pain. 2003; 101(1): 199-208.

67. Taniguchi N, Kanai S, Kawamoto M, Endo H, Higashino H. Study on application of static magnetic field for adjuvant arthritis rats. Evid Based Complement Alternat Med. 2004; 1(2): 187-91.

68. Zaringhalam J, Manaheji H, Mghsoodi N, Farokhi $\mathrm{B}$, Mirzaiee V. Spinal $\mu$-opioid receptor expression and hyperalgesia with dexamethasone in chronic adjuvantinduced arthritis in rats. Clin Exp Pharmacol Physiol. 2008; 35(11): 1309-15.

69. Chover-Gonzalez AJ, Harbuz MS, Tejedor-Real P, Gibert-Rahola J, Larsen PJ, Jessop DS. Effects of stress on susceptibility and severity of inflammation in adjuvant-induced arthritisa. Ann N Y Acad Sci. 1999; 876(1): 276-86.

70. LeHoang P, Sterkers M, Thillaye B, De Kozak Y, Coscas G, Faure JP. Primate model of uveoretinitis and vasculitis/experimental autoimmune uveoretinitis induced in cynomolgus monkeys by retinal s antigen. Ophthalmic Res. 2008; 40(3-4): 181-8.

71. Guex-Crosier Y, Raber J, Chan C-C, Kriete MS, Benichou J, Pilson RS, et al. Humanized antibodies against the alpha-chain of the IL-2 receptor and against the beta-chain shared by the IL- 2 and IL-15 receptors in a monkey uveitis model of autoimmune diseases. $\mathrm{J}$ Immunol. 1997; 158(1): 452-8.

72. Tekieh E, Manaheji H, Zeinalzadeh E. Role of serum interleukin-6 level on hyperalgesia and spinal muopioid receptor expression during the complete Freund's adjuvant-induced chronic inflammation. Kaums Journal (FEYZ). 2012; 16(3): 196-204.

73. Malheiros JM, Amaral C, Leslie ATS, Guinsburg R, Covolan L. An experimental model of neonatal nociceptive stimulation in rats. Bio Protocol. 2014; 4(21): $1-5$.

74. van de Velde F, Lourenço ND, Pinheiro HM, Bakker M. Carrageenan: a food-grade and biocompatible support for immobilisation techniques. Adv Synth Catal. 2002; 344(8): 815-35.

75. Henriques MG, Silva PM, Martins MA, Flores CA, Cunha FQ, Assreuy-Filho J, et al. Mouse paw edema. a new model for inflammation? Braz. J. Med. Biol. Res. 1986; 20(2): 243-9.

76. Sugishit AE, Amagaya S, Ogihara Y. Antiinflammatory testing methods: comparative evaluation of mice and rats. J Pharmacobio-Dyn. 1981; 4(8): 565-75.
77. Rosen A, Lundeberg T, Bytner B, Nylander I. Central changes in nociceptin dynorphin B and Met-enkephalinArg-Phe in different models of nociception. Brain Res. 2000; 857(1): 212-8.

78. Radhakrishnan R, Bement MKH, Skyba D, Sluka KA, Kehl LJ. Models of muscle pain: carrageenan model and acidic saline model. Curr Protoc Pharmacol. 2004; 5(35): doi: 10.1002/0471141755.ph0535s25.

79. Porto G-G, Vasconcelos B, Silva-Junior V-A, Souza Andrade E. The use of carrageenan for limiting the mandibular movement in rats: A preliminary experimental study. Med Oral Patol Oral Cir Bucal. 2010; 15(4): 653-7.

80. Cuzzocrea S, Sautebin L, De Sarro G, Costantino G, Rombolà L, Mazzon E, et al. Role of IL-6 in the pleurisy and lung injury caused by carrageenan. J Immunol. 1999; 163(9): 5094-104.

81. Rosa MD, Willoughby D. Screens for antiinflammatory drugs. J Pharm Pharmacol. 1971; 23(4): 297-8.

82. Di Rosa M, Giroud J, Willoughby D. Studies of the mediators of the acute inflammatory response induced in rats in different sites by carrageenan and turpentine. $\mathrm{J}$ Patho. 1971; 104(1): 15-29.

83. Lyons CR. The role of nitric oxide in inflammation. Adv Immunol. 1995; 60: 323-71.

84. Yao Z-a, Xu L, Wu H-g. Immunomodulatory function of $\kappa$-carrageenan oligosaccharides acting on LPS-activated microglial cells. Neurochem Res. 2014; 39(2): 333-43.

85. Choi H-S, Roh D-H, Yoon S-Y, Moon J-Y, Choi S-R, Kwon S-G, et al. Microglial interleukin-1 $\beta$ in the ipsilateral dorsal horn inhibits the development of mirror-image contralateral mechanical allodynia through astrocyte activation in a rat model of inflammatory pain. Pain. 2015; 156(6): 1046-59.

86. Schreiber KL, Beitz AJ, Wilcox GL. Activation of spinal microglia in a murine model of peripheral inflammation-induced, long-lasting contralateral allodynia. Neurosci Lett. 2008; 440(1): 63-7.

87. Handy RLC, Moore PK. A comparison of the effects of L-NAME, 7-NI and L-NIL on carrageenan-induced hindpaw oedema and NOS activity. Br J Pharmacol. 1998; 123(6): 1119-26.

88. Omote K, Hazama K, Kawamata T, Kawamata M, 
Nakayaka Y, Toriyabe M, et al. Peripheral nitric oxide in carrageenan-induced inflammation. Brain Res. 2001; 912(2): 171-5.

89. Janero DR. Malondialdehyde and thiobarbituric acidreactivity as diagnostic indices of lipid peroxidation and peroxidative tissue injury. Free Radic Biol Med. 1990; 9(6): 515-40.

90. Siomek A. NF- $\kappa$ B signaling pathway and free radical impact. Acta Biochim Pol. 2012; 59(3): 323-31.

91. Moyana T, Lalonde J. Carrageenan-induced intestinal injury: possible role of oxygen free radicals. Ann Clin Lab Sci. 1991; 21(4): 258-63.

92. Yang Y, Yu T, Lian Y-j, Ma R, Yang S, Cho JY. Nitric oxide synthase inhibitors: a review of patents from 2011 to the present. Expert Opin Ther Pat. 2015; 25(1): 49-68.

93. Watanabe $\mathrm{K}$, Inai $\mathrm{S}$, Jinnouchi $\mathrm{K}$, Bada $\mathrm{S}$, Hess A, Michel O, et al. Nuclear-factor kappa B (NF-kappa B)inducible nitric oxide synthase (iNOS/NOS II) pathway damages the stria vascularis in cisplatin-treated mice. Anticancer Res. 2001; 22(6C): 4081-5.

94. Sakaguchi Y, Shirahase H, Kunishiro K, Ichikawa A, Kanda M, Uehara Y. Synergistic effect of nitric oxide synthase and cyclooxygenase inhibitors on carrageenaninduced paw edema in rats. Arzneimittelforschung. 2006; 56(10): 695-9.

95. Meller S, Cummings C, Traub R, Gebhart G. The role of nitric oxide in the development and maintenance of the hyperalgesia produced by intraplantar injection of carrageenan in the rat. Neuroscience. 1994; 60(2): 367-74.

96. Gautam M, Kumar R, Prasoon P, Ray SB. Antinociceptive effect of $1400 \mathrm{~W}$, an inhibitor of inducible nitric oxide synthase, following hind paw incision in rats. Nitric Oxide. 2015; 50: 98-104.

97. Huang SS, Chiu CS, Chen HJ, Hou WC, Sheu $\mathrm{MJ}$, Lin YC, et al. Antinociceptive activities and the mechanisms of anti-inflammation of asiatic Acid in mice. Evid Based Complement Alternat Med. 2011; 2011: 1-10. doi:10.1155/2011/895857.

98. Wakabayashi K, Inagaki T, Fujimoto Y, Fukuda Y. Induction by degraded carrageenan of colorectal tumors in rats. Cancer Lett. 1978; 4: 171-6.

99. Trentham DE, Townes AS, Kang AH. Autoimmunity to type II collagen an experimental model of arthritis. J Exp Med. 1977; 146(3): 857-68.

100. Elliott MJ, Maini RN, Feldmann M, Long-Fox A, Charles P, Katsikis P, et al. Treatment of rheumatoid arthritis with chimeric monoclonal antibodies to tumor necrosis factor $\alpha$. Arthritis Rheum. 1993; 36(12): 1681-90.

101. Patro N, Sharma A, Kariaya K, Patro I. Spirulina platensis protects neurons via suppression of glial activation and peripheral sensitization leading to restoration of motor function in collagen-induced arthritic rats. Indian J Exp Biol. 2011; 49: 739-48.

102. Holmdahl R, Andersson ME, Goldschmidt TJ, Jansson L, Karlsson M, Malmstrom V, et al. Collagen induced arthritis as an experimental model for rheumatoid arthritis. APMIS. 1989; 97(7-12): 575-84.

103. Holmdahl R, Jansson L, Larsson E, Rubin K, Klareskog L. Homologous type II collagen induces chronic and progressive arthritis in mice. Arthritis Rheum. 1986; 29(1): 106-13.

104. Sato M, Sano H, Iwaki D, Kudo K, Konishi M, Takahashi H, et al. Direct binding of Toll-like receptor 2 to zymosan, and zymosan-induced NF- $\kappa \mathrm{B}$ activation and TNF- $\alpha$ secretion are down-regulated by lung collectin surfactant protein A. J Immunol. 2003; 171(1): 417-25.

105. Gemmell DK, Cottney J, Lewis AJ. Comparative effects of drugs on four paw oedema models in the rat. Agents Actions. 1979; 9(1): 107-16.

106. Damas J, Remacle-Volon G. Mast cell amines and the oedema induced by zymosan and carrageenans in rats. Eur J Pharmacol. 1986; 121(3): 367-76.

107. Meller S, Gebhart G. Intraplantar zymosan as a reliable, quantifiable model of thermal and mechanical hyperalgesia in the rat. Eur J Pain. 1997; 1(1): 43-52.

108. Converse AK, Larsen EC, Engle JW, Barnhart TE, Nickles RJ, Duncan ID. 11C-(R)-PK11195 PET imaging of microglial activation and response to minocycline in zymosan-treated rats. J Nucl Med. 2011; 52(2): 257-62.

109. Harrigan TJ, Abdullaev IF, Jourd'heuil D, Mongin AA. Activation of microglia with zymosan promotes excitatory amino acid release via volume-regulated anion channels: the role of NADPH oxidases. J Neurochem. 2008; 106(6): 2449-62. 